\title{
Particle Distributions and Noise in Metal Particle Tapes
}

\author{
Mustafa M. Aziz, Barry K. Middleton, and Jim J. Miles
}

\begin{abstract}
The magnetization spectrum from an erased (ac demagnetized or dc saturated) particulate medium was derived analytically using the autocorrelation function of a long line of separate and contiguous particles. The derived expressions indicated a strong dependence on the percentage of chains and their length inside the magnetic medium. Using the values of these quantities as fitting parameters, good agreement to measured noise spectra from a digital tape system was obtained.
\end{abstract}

Index Terms-Metal particle, noise, tape.

\section{INTRODUCTION}

A DVANCED tape media consist of needle shaped iron particles of approximately $100-\mathrm{nm}$ lengths, axial ratios of around 8 and coated with a protective oxide layer of thickness between 2 and $4 \mathrm{~nm}$ [1], [2]. These particles are aligned during manufacture by an external magnetic field in the direction of the tape motion to increase the magnitude of the replay signal. However, the alignment process is not perfect and leads to a distribution of particle axes throughout the tape. Furthermore, particles also exhibit variation in their sizes throughout the volume of the tape. These variations constitute noise and conspire to contaminate the replay output signal and limit the recording resolution of magnetic tapes. This paper is concerned with the noise produced by such media.

By treating the individual particles as independent voltage sources the replay power spectral density of a tape medium has previously been predicted as the sum of these individual voltages [3]-[5]. The effects of the variations in particle attributes were included subject to their statistical distributions. Simplified models of clustering in particulate media were also produced and yielded good agreement with measured noise spectra [6], [7]. These models used size distributions that did not allow closed form expressions for the noise spectra to be derived, and treated size variations and particle chaining independently to explain the measurements.

This paper utilizes the autocorrelation method to derive an analytical expression for the magnetization power spectral density of an erased (ac demagnetized or dc saturated) particulate medium [8] taking into account particle size variations and chaining to explain measurements on ac-demagnetized tapes.

Manuscript received February 11, 2002; revised May 23, 2002. This work was supported by the Engineering and Physical Sciences Research Council under Grant GR/M46648/01.

M. M. Aziz is with the School of Engineering and Computer Science, University of Exeter, Exeter EX4 4QF, U.K. (e-mail: M.M.Aziz@exeter.ac.uk).

B. K. Middleton and J. J. Miles are with the Department of Computer Science, University of Manchester, Manchester M13 9PL, U.K. (e-mail: B.Middleton@cs.man.ac.uk, J.Miles@man.ac.uk).

Digital Object Identifier 10.1109/TMAG.2002.802828.

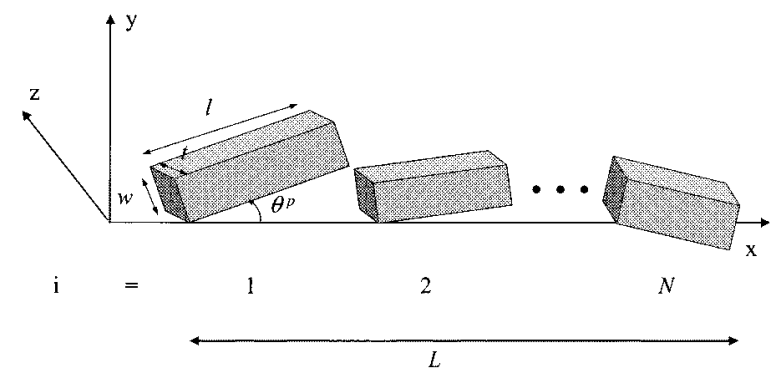

Fig. 1. Line of particles.

\section{THEORY}

Fig. 1 illustrates a line of particles of length $L$ containing $N$ individual particles. Each particle has a length $l$, orientation $\theta^{p}$, width $w$, and thickness $t$ and, hence a volume $\vartheta^{p}=w t l$.

\section{A. Uncorrelated Particles}

With no correlations between the particles, it was shown that for small uniform distributions of particle orientations of the order of $\left|\theta^{p}\right|<\tan ^{-1}(w / l)$ where $l>w$, the magnetiation power spectral density is given by [8]:

$$
S_{p}(k)=\frac{2 \overline{\left(\mathbf{M}^{p}\right)^{2}}}{k^{2}} D \bar{t} \bar{w} \cdot \overline{(1-\cos (k l))}
$$

where $\mathbf{M}^{p}$ is the uniform magnetization vector inside each particle, $D$ is the particle density, $\bar{t}$ and $\bar{w}$ are the average particle width and thickness respectively, and the overbar indicates ensemble averages. $k=2 \pi / \lambda$ is the wavenumber and $\lambda$ is the wavelength.

Assuming that the particle lengths follow the Gamma distribution [8]:

$$
f(l)=\frac{l^{\left(\bar{l}^{2} / \sigma^{2}-1\right)} e^{\left(-\bar{l} / \sigma^{2}\right)}}{\left(\frac{\sigma^{2}}{\bar{l}}\right)^{\left(\bar{l}^{2} / \sigma^{2}\right)} \Gamma\left(\frac{\bar{l}^{2}}{\sigma^{2}}\right)}
$$

where $\sigma$ is the standard deviation of the particle length, $\bar{l}$ is the average particle length, and $\Gamma($.$) is the Gamma function, then$ the averages in (1) can be evaluated analytically to yield

$$
S_{p}(k)=\frac{2 \overline{\left(\mathbf{M}^{p}\right)^{2}}}{k^{2}} \cdot D \bar{t} \bar{w} \cdot\left\{1-\frac{\cos \left[\left(\frac{\bar{l}^{2}}{\sigma^{2}}\right) \tan ^{-1}\left(\frac{k \cdot \sigma^{2}}{\bar{l}}\right)\right]}{\left[1+\left(\frac{k \cdot \sigma^{2}}{\bar{l}}\right)^{2}\right]^{\left(\bar{l}^{2} / \sigma^{2}\right) / 2}}\right\} .
$$

\section{B. Chains of Particles}

Observations of arrangements of carbon steel wires in a vinyl chloride binder in a large scale model of a tape coating have 


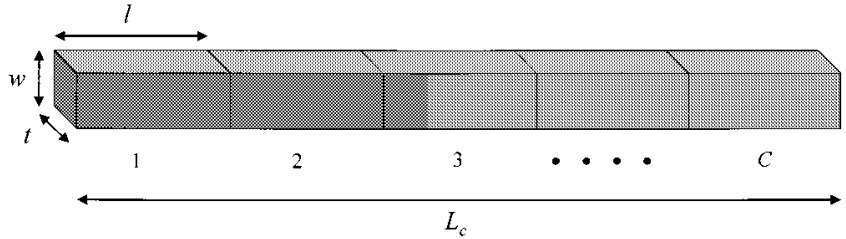

Fig. 2. Chain of particles.

indicated the presence of strong interactions between particles and the formation of long chains of particles during alignment for packing fractions greater than 0.01 [9]. The formation of chains was also observed in computer simulations of elongated Fe particles in a strong applied field [10]. Hence, correlations in a particulate medium will be simulated using chains of contiguous particles as demonstrated in Fig. 2, with uniform magnetization throughout each chain.

To simplify the modeling and the fitting process, it is assumed that each chain will contain $C$ identical particles and the length of each chain is therefore equal to $L_{c}=C \bar{l}$.

The power spectral density of a line of particles containing individual and contigous particles, both assumed uncorrelated, is given by [8]

$$
\begin{aligned}
& S(k)=\frac{2 \overline{\left(\mathbf{M}^{p}\right)^{2}}}{k^{2}} \\
& \cdot D \bar{t} \bar{w}\{(1-F \bar{C}) \overline{(1-\cos (k l))}+F \overline{(1-\cos (k C \bar{l}))}\}
\end{aligned}
$$

where

$$
F=\lim _{N \rightarrow \infty} \frac{N_{c}}{N}
$$

is the proportion of chains inside the medium and $N_{c}$ is the number of chains in the medium. For a uniform distribution of particle numbers inside each chain between 1 and a maximum of $C_{m}$, inclusive, the magnetization power spectral density was found to be

$$
\begin{aligned}
S(k)= & \frac{2 \overline{\left(\mathbf{M}^{p}\right)^{2}}}{k^{2}} \\
& \cdot D \bar{t} \bar{w}\left\{\left(1-\frac{F\left(C_{m}+1\right)}{2}\right)\right. \\
& \cdot\left[1-\frac{\cos \left[\left(\frac{\bar{l}^{2}}{\sigma^{2}}\right) \tan ^{-1}\left(\frac{k \sigma^{2}}{\bar{l}}\right)\right]}{\left.\left[1+\left(\frac{k \cdot \sigma^{2}}{\bar{l}}\right)^{2}\right]^{\left(\bar{l}^{2} / \sigma^{2}\right) / 2}\right]}\right. \\
& \left.+F\left[1-\frac{\cos \left(\frac{k \bar{l}\left(C_{m}+1\right)}{2}\right) \sin \left(\frac{k \bar{l} C_{m}}{2}\right)}{C_{m} \sin \left(\frac{k \bar{l}}{2}\right)}\right]\right\} .
\end{aligned}
$$

\section{EXPERIMENTAL MEASUREMENTS AND INTERPRETATION}

The noise measurements were performed on a helical scan digital tape system using ac-demagnetized, 4-mm metal particle tapes. The properties of the tapes are given in Table I. A vibrating-sample magnetometer was used to measure the hysteresis loop (shown in Fig. 3) of a tape sample to obtain the magnetic properties of the tapes. The magnetic layer thickness and particle packing fraction values of the tapes were provided by the tape manufacturer.
TABLE I

Metal Particle Tape Properties

\begin{tabular}{ccccc}
\hline $\begin{array}{c}\text { Coercivity } \\
(\mathrm{kA} / \mathrm{m})\end{array}$ & $\begin{array}{c}\text { Remanent } \\
\text { magnetisation } \\
(\mathrm{kA} / \mathrm{m})\end{array}$ & Squareness & $\begin{array}{c}\text { Magnetic layer } \\
\text { thickness }(\mu \mathrm{m})\end{array}$ & $\begin{array}{c}\text { Particle packing } \\
\text { fraction }\end{array}$ \\
\hline 185 & 270 & 0.9 & 0.1 & 36 to $39 \%$ \\
\hline
\end{tabular}

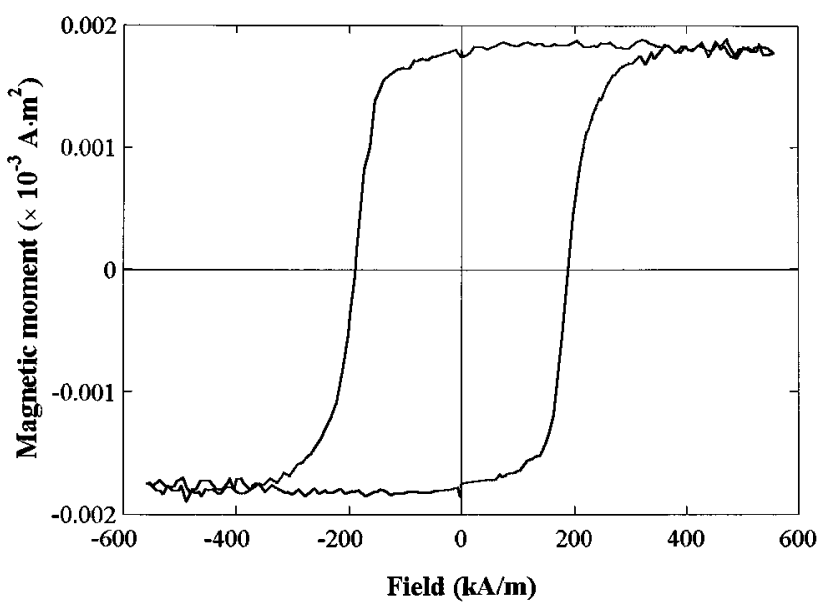

Fig. 3. Hysteresis loop of metal particle tape sample measured in the direction of the longitudinal tape motion and at room temperature.

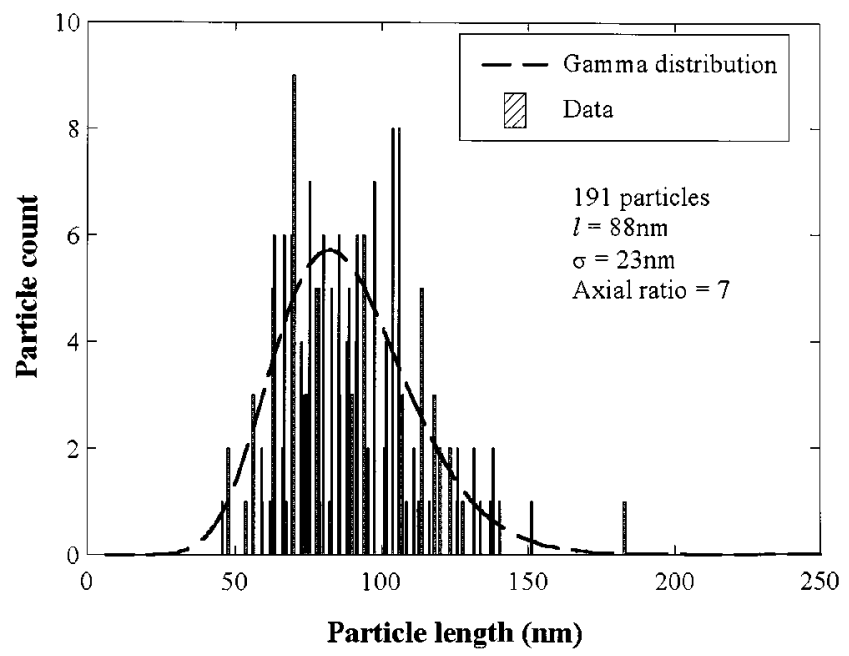

Fig. 4. Particle length counts of 191 particles from a metal power sample compared with the calculated Gamma distribution. Particles have an average length of $88 \mathrm{~nm}$, standard deviation $23 \mathrm{~nm}$, and an axial ratio of 7 .

For comparison with spectral measurements, information is required about the distribution of particle sizes inside a typical metal particle tape. This is provided by the data in Fig. 4, which shows a particle length distribution obtained from an experimental metal powder sample with coercivity $197 \mathrm{kA} / \mathrm{m}$ and saturation magnetization $143 \mathrm{Am}^{2} / \mathrm{kg}$ [1]. The Gamma distribution is also plotted in Fig. 4 using (2) for comparison using the measured average particle length of $88 \mathrm{~nm}$ and standard deviation of $23 \mathrm{~nm}$.

In the following fittings, the values of the average particle length and axial ratio of the particles will be those shown in Fig. 4, as they represent values of an advanced metal particle tape medium similar to the tape medium used in the measurements. 


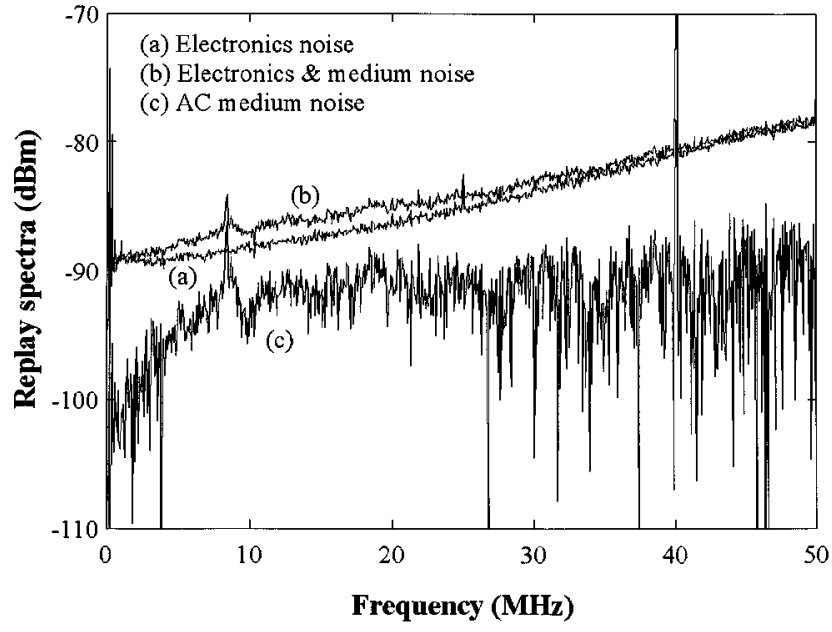

Fig. 5. Measured replay noise spectra from an ac-demagnetized tape.

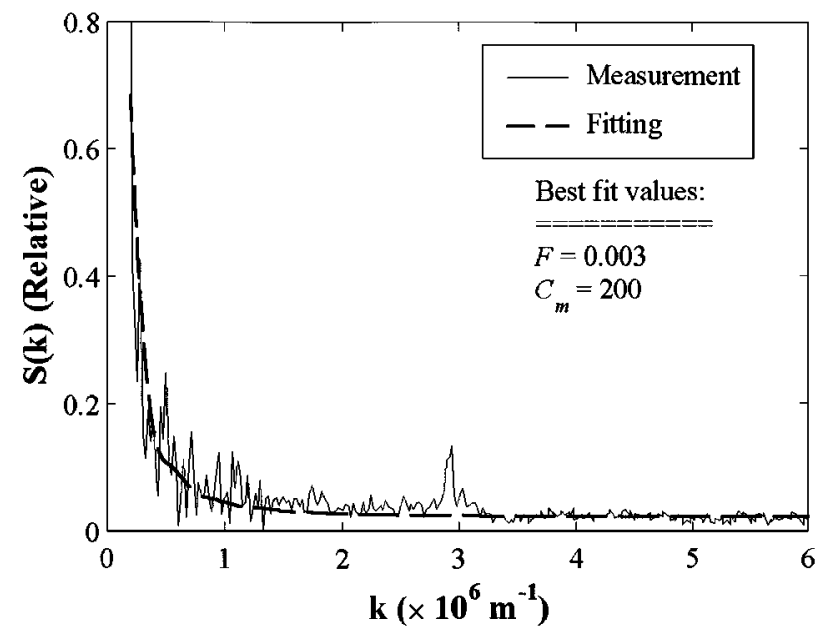

Fig. 6. Measured medium noise spectrum normalized by the replay losses and the fitted magnetisaton power spectral density given in (5).

Replay was achieved using an inductive head with gap length $0.2 \mu \mathrm{m}$. The rotating head was moving at a speed of $18 \mathrm{~m} / \mathrm{s}$ and the tape was moving at $0.023 \mathrm{~m} / \mathrm{s}$, with a flying height of approximately $40 \mathrm{~nm}$. A spectrum analyzer was used to capture and analyze the replay signals with the following settings: bandwidth $50 \mathrm{MHz}$, resolution bandwidth $10 \mathrm{kHz}$, and video bandwidth $10 \mathrm{kHz}$, giving a sweep time of $1.272 \mathrm{~s}$. The captured replay spectra were the average of 64 accumulated sweeps.

Initially, the voltage from the replay head was measured in the absence of the tape to give the system noise spectrum (electronics plus drum noise) as is shown by curve (a) in Fig. 5. The spectrum was then measured in the presence of the moving virgin tape to yield the total noise spectrum of curve (b). Subtracting, as powers, the electronics noise in (a) from the total measured noise spectrum in (b) yields the medium noise power spectral density of curve (c).
The medium noise spectrum was then normalized by the replay loss factors (gap-, spacing-, and thickness-loss factors) using the Lindholm head field transforms given in [11]. The resulting spectrum, shown in Fig. 6, represents the particle magnetization's power spectral density.

Using a least-squares algorithm, (5) was fitted to the measured magnetization spectrum with the constant of proportionality, the chain percentage $F$, and maximum chain length $C_{m}$ as the fitting parameters. Fig. 6 shows the results of the fitting where it can be seen that a chain percentage of $0.3 \%$ and a maximum chain length of 200 particles yielded good agreement between the measured and calculated spectra over a wide range of wavelengths.

\section{CONCLUSION}

Closed form expressions for the medium noise spectra in particulate media have been derived which include particle chains. The comparison of (5) with measurement has indicated the dependence of the shape of the medium magnetization spectrum on the chain length $C$ and proportion $F$ inside the medium especially at long wavelengths.

\section{ACKNOWLEDGMENT}

The authors wish to thank M. del Puerto Morales Herrero of the Materials Science Institute of Madrid for providing the data of the particle length counts. The authors would also like to thank the referees of this paper for their helpful comments.

\section{REFERENCES}

[1] M. P. Morales, S. A. Walton, L. S. Prichard, C. J. Serna, D. P. E. Dickson, and K. O'Grady, "Characterization of advanced metal particle recording media pigments," J. Magn. Magn. Mater, vol. 190, pp. 357-370, 1998.

[2] M. P. Sharrock, "Recent advances in metal particulate recording media, toward the ultimate particle," IEEE Trans. Magn., vol. 36, pp. $2420-2425,2000$

[3] I. Stein, "Analysis of noise from magnetic storage media," J. Appl. Phys., vol. 34, no. 7, pp. 1976-1990, 1963.

[4] L. Thurlings, "Statistical analysis of signal and noise in magnetic recording," IEEE Trans. Magn., vol. MAG-16, pp. 507-513, 1980.

[5] H. N. Bertram, Theory of Magnetic Recording. Cambridge, UK: Cambridge Unive. Press, 1994.

[6] P. Luo and H. N. Bertram, "Tape medium noise measurements and analysis," IEEE Trans. Magn., vol. 37, pp. 1620-1623, 2002.

[7] L. L. Nunnelley, D. E. Heim, and T. C. Arnoldussen, "Flux noise in particulate media: Measurement and interpretation," IEEE Trans. Magn., vol. MAG-23, pp. 1767-1775, 1987.

[8] M. M. Aziz, B. K. Middleton, and J. J. Miles, "Autocorrelation analysis of the particle magnetization in erased particulate media," IEEE Trans. Magn., vol. 38, pp. 279-287, 2002.

[9] M. Matsumoto, "A study of particle arrangement in magnetic tapes," IEEE Trans. Magn., vol. MAG-15, pp. 1312-1314, 1979.

[10] G. N. Coverdale, R. W. Chantrell, G. A. R. Martin, A. Bradbury, A. Hart, and D. A. Parker, "Cluster analysis of the microstructure of colloidal dispersions using the maximum entropy technique," J. Magn. Magn. Mater, vol. 188, pp. 41-51, 1998.

[11] D. A. Lindholm, "Dependence of reproducing gap null on head geometry,” IEEE Trans. Magn., vol. MAG-11, pp. 1692-1696, 1975. 